OPEN ACCESS

Edited by:

Roberta Tasso,

University of Genoa, Italy

Reviewed by:

Emanuele Cocucci,

The Ohio State University,

United States

Flora Guerra

University of Salento, Italy

*Correspondence:

Yu-Hui Shen

syh10994@rih.com.cn

Yan-Jun Liu

Yanjun_Liu@fudan.edu.cn

tORCID:

Yu-Hui Shen

orcid.org/0000-0002-9220-6473

Yan-Jun Liu

orcid.org/0000-0001-6535-8431

${ }^{\ddagger}$ These authors have contributed equally to this work and share first authorship

Specialty section:

This article was submitted to Molecular and Cellular Oncology, a section of the journal Frontiers in Oncology

Received: 13 May 2021 Accepted: 12 August 2021 Published: 30 August 2021

Citation:

Xu Y-Q, Bao Q-Y, Yu S-X, Liu Q, Xie Y,

Li X, Liu Y-J and Shen Y-H (2021) A Novel Microfluidic Chip for Fast,

Sensitive Quantification of Plasma Extracellular Vesicles as Biomarkers in Patients With Osteosarcoma.

Front. Oncol. 11:709255. doi: 10.3389/fonc.2021.709255

\section{A Novel Microfluidic Chip for Fast, Sensitive Quantification of Plasma Extracellular Vesicles as Biomarkers in Patients With Osteosarcoma}

\author{
Yi-Qi Xu ${ }^{1 \neq}$, Qi-Yuan Bao ${ }^{1 \neq}$, Sai-Xi Yu ${ }^{2}$, Qi Liu ${ }^{1}$, Yan Xie ${ }^{3}, X_{i n} L^{3}$, Yan-Jun Liu ${ }^{2 * t}$ and \\ Yu-Hui Shen ${ }^{1 * T}$ \\ ${ }^{1}$ Department of Orthopedics, Ruijin Hospital, Shanghai Jiaotong University School of Medicine, Shanghai, China, \\ 2 Shanghai Institute of Cardiovascular Diseases, Shanghai Key Laboratory of Medical Epigenetics, International Co-laboratory \\ of Medical Epigenetics and Metabolism (Ministry of Science and Technology), Institutes of Biomedical Sciences, Zhongshan \\ Hospital, Fudan University, Shanghai, China, ${ }^{3}$ Engineering Research Center for Nanophotonics and Advanced Instrument, \\ Joint Institute of Advanced Science and Technology, School of Physics and Electronic Science, East China Normal \\ University, Shanghai, China
}

Plasma circulating extracellular vesicle (EV) has emerged as a promising biomarker for diagnosis and prognosis of various epithelial tumors. However, fast and efficient capture of EVs with microfluidic chip in sarcoma remains to be established. Herein, we reported a ZnO-nanorods integrated (ZNI) microfluidic chip, where EV capture antibody was uniformly grafted to the surface of the ZnO-nanorods of the chip to enhance the plasma turbulence formation and the capture efficiency at the micro-scale. Based on osteosarcoma (OS) cell line, we demonstrated that a combination of CD81 and CD63 antibody on ZNI chip yielded the greatest amount of total EVs, with an extra sensitive limit of detection (LOD) of $\sim 10^{4}$ particles $\mathrm{mL}^{-1}$. Furthermore, the addition of fluorescent labeling of Vimentin (VIM), a previously reported sarcoma cell surface biomarker, could enabled the dual visualization of total plasma EVs and VIM-positive EVs from OS patients' plasma. Based on our ZNI chip, we found that the amount of plasma total EVs was significantly different between OS and healthy donors (1562 a.u. versus 639 a.u., $p<0.05$ ), but not between metastatic and nonmetastatic OS ( $p>0.05$ ). Interestingly, patients with metastatic disease had a significantly greater amount of VIM-positive EVs (1411 a.u. versus 231 a.u.., $\mathrm{p}<0.05)$ and increased VIM-positive/total EVs ratio $(0.943$ versus 0.211 , $\mathrm{p}<0.05$ ) in comparison with the nonmetastatic counterpart. Therefore, our ZNI microfluidic chip has great potential for the fast quantification of plasma EVs, and the microfluidic-based quantification of total and VIM-positive EVs might serve as a promising biomarker for the diagnosis and surveillance in OS patients.

Keywords: ZnO-nanorods, microfluidic chip, vimentin, osteosarcoma, extracellular vesicles 


\section{INTRODUCTION}

Liquid biopsy of circulating extracellular vesicle (EV) has nowadays gained an increasing popularity as a promising source of prognostic and therapeutic biomarkers for several common types of epithelial cancer (1-3). Tumor-derived EV has been implicated in multiple steps of cancer pathogenesis and metastasis, including the organotropic determination, microenvironment formation, immune response (4-7). Unlike other liquid-based approach such as circulating tumor cell (CTC) and cell-free tumor DNA (ctDNA), EV is reported to be actively secreted and functionally transported within blood stream, with a favorable stability and high abundance $\left(\sim 10^{10}\right.$ particles $\left./ \mathrm{mL}\right)(8-10)$, thereby regarded as a promising source of cancer biomarker.

For sarcoma, however, capturing and quantifying of circulating EVs with fast and simple tools remains to be exploited. Currently, the isolation of EVs mainly relies on ultracentrifugation (UC), which is limited by large sample consumption, low sample recovery rate and complicated procedures (11-14). Other methods such as ultra-filtration and precipitation-based commercial kits suffer from poor standardization and the contamination of non-vesicular particles (15-18). In recent years, microfluidic chips with nanostructure have been increasingly accepted as a promising tool for the separation and detection of EVs in a high-throughput and integrable manner (19-23). Nanomaterials have strong adsorption capacity of EVs owing to their high surface area to volume ratio and reduced near-surface flow stagnation, thereby enhancing the combination of vesicles and the surface of nanomaterials $(3,24$, 25). Among the many nanomaterials, $\mathrm{ZnO}$-nanorods are especially advantageous in biological detection due to their low production cost, easy preparation, robustness, and biocompatibility (26-28). Furthermore, large specific surface area, interface electron transfer effect and optical waveguide effect of $\mathrm{ZnO}$-nanorods could significantly enhance the fluorescence signal (29-31).

Herein, we designed a $\mathrm{ZnO}$-nanorods integrated (ZNI) microfluidic chip (Figure 1). Under the synergistic effect of the herringbone $(\mathrm{HB})$ structure and $\mathrm{ZnO}$ nanorods, the formation of turbulence of plasma flowing through the chip increased the collision probability of EVs with nanorods, resulting in a favorable capture efficiency at the micro-scale $(22,32,33)$. Next, we performed a series of optimization and validation study using EVs from cell line and patient plasma of osteosarcoma, the most common bone sarcoma. We found that our device had an extremely low limit of detection (LOD) of $\sim 10^{4}$ particles $/ \mathrm{mL}$, which was drastically below the EV concentration in plasma. Furthermore, our microfluidic-based EV quantification could not only distinguish osteosarcoma patients from healthy donors, but also the metastatic diseases from the non-metastatic counterparts.

\section{METHODS AND MATERIALS}

\section{Preparation of Microfluidic Device}

The $\mathrm{ZnO}$ growth chip and the ZNI chip were fabricated using a standard soft lithography process. The SU-8 2050 photoresist (MicroChem) was spin-coated on the silicon wafer. Following ultraviolet (UV) exposure and development, the mold was treated with trimethylchlorosilane and then filled with PDMS (RTV615) prepolymer at a 10:1 ratio of base polymer to crosslinker. Next, the mold filled with PDMS prepolymer was debubbled in a vacuum manner. The PDMS replicas were peeled off after curing at $80^{\circ} \mathrm{C}$ for 2 hours. The $\mathrm{ZnO}$ growth chip consisted of 4 channels with a width of $200 \mu \mathrm{m}$ and a height of $75 \mu \mathrm{m}$, and the ZNI chip consisted of the main microfluidic channel (height: 10 , 20,30, $40 \mu \mathrm{m}$ ) and the HB structure (height: $5 \mu \mathrm{m}$ ) (Figure 2B).

\section{Preparation and Functionalization of ZnO-Nanorods on ZNI Chip}

The seed solution, $10 \mathrm{mM}$ zinc acetate ethanol solution, was spin-coated on the clean glass slide at a rotation speed of $2000 \mathrm{r} /$ min, and spin-coating was repeated three times. The glass slide was placed on a heating platform at $300^{\circ} \mathrm{C}$ for $2 \mathrm{~h}$ to complete the preparation of seed layer. Then, $0.2 \mathrm{~g}$ polyethyleneimine (PEI) $(12.5 \mathrm{mM}), 0.372 \mathrm{~g} \mathrm{Zn}\left(\mathrm{NO}_{3}\right)_{2} \cdot 6 \mathrm{H}_{2} \mathrm{O}(25 \mathrm{mM}), 0.0875 \mathrm{~g}$ hexamethylenetetramine (HMTA) (12.50 mM) were dissolved in deionized water in a $50 \mathrm{~mL}$ volumetric flask to prepare $\mathrm{ZnO}$ growth solution, and $\mathrm{NH}_{3} \cdot \mathrm{H}_{2} \mathrm{O}$ was added to adjust the $\mathrm{pH}$ to 10.60. The $\mathrm{ZnO}$ growth chip was bonded to the glass slide covered with the seed layer, the growth solution was added to the reservoir, and the syringe pump was used to pump the growth solution at a speed of $4.8 \mu \mathrm{L} / \mathrm{min}$. $\mathrm{ZnO}$ nanorods were prepared by local heating at a temperature of $90^{\circ} \mathrm{C}$ for $3 \mathrm{~h}$, which was the optimized parameter for acquiring the largest binging surface area of protein accordingly to our previous work (30). Then, the $\mathrm{ZnO}$ growth chip was peeled off and the ZNI chip with $\mathrm{HB}$ structure was bonded to the glass slide with $\mathrm{ZnO}$-nanorods to complete the final device assembly (Figures 2A, C). The morphology of $\mathrm{ZnO}$-nanorods was characterized by scanning electron microscopy (ZEISS Supra 40VP SEM) (Figures 2D, E).

To functionalize the nanorods with EV capture antibody, the surface of $\mathrm{ZnO}$-nanorods by exposure to 4\% (3-mercaptopropyl) trimethoxysilane (MPS) anhydrous ethanol solution at room temperature for $30 \mathrm{~min}$. Excess silane was washed away with anhydrous ethanol. Next, $0.25 \mathrm{mM} \mathrm{N}$-maleimidobutyryloxysuccinimide ester (GMBS) DMSO solution was injected into the ZNI chip to modify the surface of nanorods for 40 min. After washing with PBS, $10 \mu \mathrm{g} / \mathrm{mL}$ Protein G PBS solution was fed into the chip to coat the surface for $1 \mathrm{~h}$ at $4^{\circ} \mathrm{C}$ (34). After washing with PBS, $10 \mu \mathrm{g} / \mathrm{mL}$ anti-CD63 and anti-CD81 mixed antibody PBS solution was pumped into the chip channel for $1 \mathrm{~h}$ at $4{ }^{\circ} \mathrm{C}$. At the end, the chip was blocked with $3 \%$ BSA for $30 \mathrm{~min}$ and stored at $4^{\circ} \mathrm{C}$ before the experiments (Figure S1). Fourier Transform infrared spectroscopy (FT-IR) was used to detect the relevant chemical groups in each step of the antibody covalent coupling (Figure S2). The CD63+CD81 double-antibody was characterized by fluorescently labeled goat anti-mouse IgG (Figure S3). The information of the materials and reagents used in this research were displayed in Table S1.

\section{Collection of Plasma EVs}

The study protocol was approved by the ethical committee of the institution according to relevant guidelines and was prospectively collected in our longitudinal observational clinical research project registered at clinicaltrial.gov (trial ID: NCT03108677). OS patients 


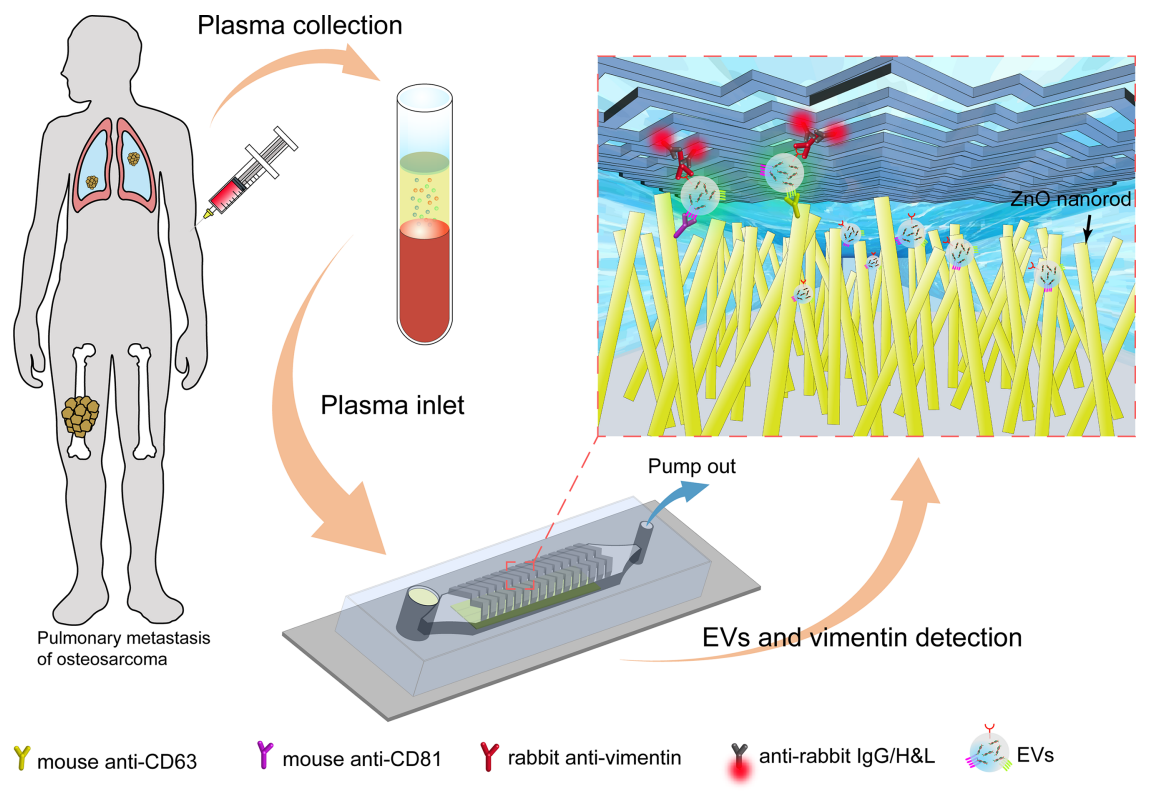

FIGURE 1 | The overall process flow of the ZnO nanorods integrated (ZNI) microfluidic chip for detection of EVs from osteosarcoma patients. First, the peripheral blood of osteosarcoma patients was collected, and the blood cells and plasma were separated by centrifugation. At the other end of the chip, a syringe pump was used to pump the plasma in the sample reservoir at a fixed speed to capture EVs in the plasma. Next, the lipophilic membrane dye 3,3'-dioctadecyloxacarbocyanine (DiO) was used to quantify the captured EVs in the chip. Finally, EV membrane vimentin (VIM, a sarcoma biomarker) was detected using a second signal based on fluorescence conjugated antibodies.

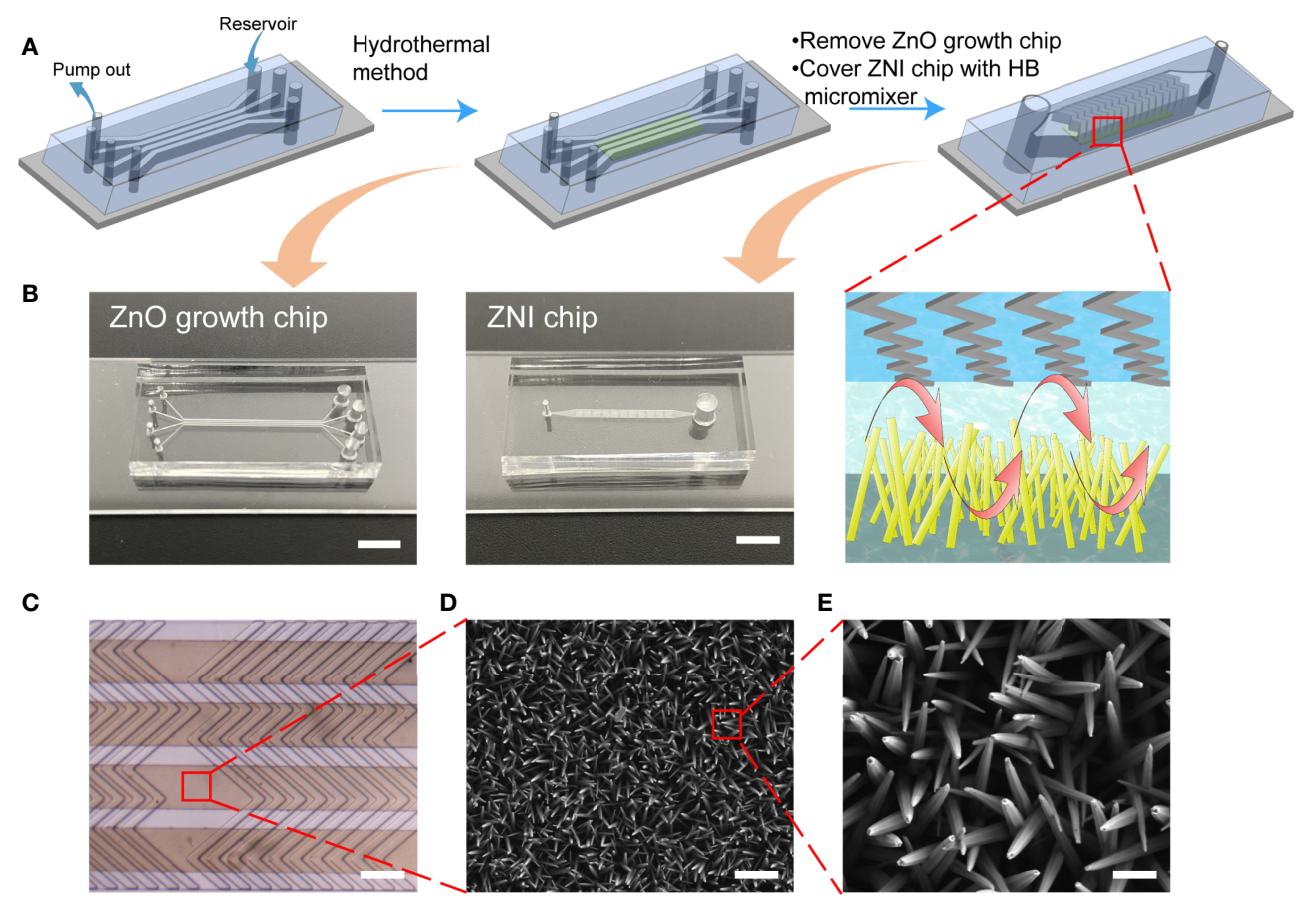

FIGURE 2 | The flow chart of manufacturing the ZNI chip. (A) The ZnO nanoarray was prepared in ZnO growth chip by a hydrothermal method. 4 groups of nanoarrays were prepared simultaneously. Then, the $\mathrm{ZnO}$ growth chip was peeled off and bonded the chip with the herringbone (HB) micromixer structure to the top of the nanoarray to complete the assembly of the device. (B) Photographic image of the ZnO growth chip and ZNI chip. Scale bar: $4 \mathrm{~mm}$. (C) Stereoscope image of ZNI chip. Scale bar: $200 \mu \mathrm{m}$. (D, E) Scanning electron microscope (SEM) micrographs of ZnO nanorods. (D) Scale bar: $1 \mu \mathrm{m}$. (E) Scale bar: $200 \mathrm{~nm}$. 
were free of any systemic treatment for at least 1 month before the blood draw to minimize the potential intra-individual variation. All plasma was collected using Vacutainer Glass Blood Collection Tubes with Acid Citrate Dextrose (BD, USA) and centrifuged at $2000 \mathrm{~g}$ and $4000 \mathrm{~g}$ respectively for $10 \mathrm{~min}$ to remove cell debris before introduced into the ZNI chip platform. For the characterization of plasma EVs, the plasma was then diluted 1:2 with PBS and filtered with the $0.45 \mu \mathrm{m}$ filter to remove the larger particles. Following ultracentrifugation at $110,000 \mathrm{~g}$ for 11 hours and another ultracentrifugation at $110,000 \mathrm{~g}$ for 70 minutes, the final EV pellets were resuspended in PBS and performed nanoparticle tracking analysis (NTA) as validation for quantification of plasma EVs.

\section{Isolation of OS Cell Line-Derived EVs}

OS cell lines were purchased from The Cell Bank of Type Culture Collection of Chinese Academy of Sciences, Shanghai, China (www. cellbank.org.cn) with corresponding STR profiling as cell-line authentication. The medium we used was EV-depleted complete medium (EDCM) consisted of: DMEM+10\% EV-depleted FBS+1\% P-S. $45 \mathrm{~mL}$ of culture supernatant from osteosarcoma cell lines HOS, $143 \mathrm{~B}, \mathrm{U} 2 \mathrm{OS}$, and MG63 was centrifuged at 2,000 $\mathrm{g}$ for $15 \mathrm{~min}$ under $4^{\circ} \mathrm{C}$ to remove cell debris. The obtained supernatant was then centrifuged at $10,000 \mathrm{~g}$ for $30 \mathrm{~min}$ under $4^{\circ} \mathrm{C}$ and filtering with the $0.45 \mu \mathrm{m}$ filter to remove the remaining cell debris and microvesicles. Next, the supernatant was centrifuged at $100,000 \mathrm{~g}$ for $90 \mathrm{~min}$, and the precipitate was resuspended in PBS followed by another ultracentrifugation at $100,000 \mathrm{~g}$ for $90 \mathrm{~min}$ under $4^{\circ} \mathrm{C}$. Finally, the precipitate was resuspended in $\mathrm{PBS}$ and stored at $-20^{\circ} \mathrm{C}$ for future western blot analysis.

\section{Characterization of EVs}

The morphology of the HOS-derived EVs was characterized using a Tecnai G2 20 TWIN transmission electron microscope (TEM). $2 \mu \mathrm{L}$ of EV pellet was loaded on a 400 -mesh carboncoated copper grid and then negatively stained with $2 \%$ phosphotungstic acid for $10 \mathrm{~min}$. After removal of the excess dyes, the prepared sample was left to dry at room temperature and observed under a voltage of $200 \mathrm{kV}$. For characterization of the captured EVs, the EVs immobilized on $\mathrm{ZnO}$ nanorods were fixed in $4 \%$ paraformaldehyde (PFA) for $1 \mathrm{~h}$. The samples were dehydrated by sequential immersion in $30,50,75,85,95$, and $100 \%$ ethanol solutions for $10 \mathrm{~min}$ per solution. After overnight lyophilization, sputter-coating with gold was performed at room temperature. The morphology of EVs immobilized on $\mathrm{ZnO}$ nanorods was then observed using SEM. To quantify the EV amount and size distribution, isolated EVs were proceeded with nanoparticle tracking analysis (NTA) as a gold standard. The videos of 60 -sec duration taken by its camera $0.743 \mu \mathrm{m} / \mathrm{px}$ are analyzed with the Software (ZetaView 8.04.02).

\section{EV Capture and Quantification Using ZNI Chip}

$100 \mu \mathrm{l}$ HOS-derived EV suspension, which was used to optimize the functional parameters, was pumped through the ZNI chip coated with anti-CD63 and CD81 antibodies at a flow rate of $2 \mu \mathrm{L} / \mathrm{min}$ using a micro syringe pump, and then $50 \mu \mathrm{M} \mathrm{DiO}$ membrane dye was injected into the chip at a speed of $2 \mu \mathrm{L} / \mathrm{min}$ and incubated at room temperature for $30 \mathrm{~min}$. After being rinsed three times with PBS, fluorescence microscope (Nikon Ti2-U) was used to complete the quantification of EVs. Besides, $50 \mu \mathrm{L}$ plasma (PBS diluted to $200 \mu \mathrm{L}$ ) was introduced into the ZNI chip coated with anti-CD63 and CD81 antibodies via the same procedure as above to complete $\mathrm{DiO}$ labeling of EVs. After being rinsed three times with PBS, $10 \mu \mathrm{g} / \mathrm{mL}$ rabbit anti-vimentin (VIM) antibody was pump into the chip at a speed of $1 \mu \mathrm{L} / \mathrm{min}$ for $40 \mathrm{~min}$. The chip was rinsed 3 times to wash away excess antibody, $20 \mu \mathrm{g} / \mathrm{mL}$ Alexa Fluor 647 labeled goat anti-rabbit IgG $(\mathrm{H}+\mathrm{L})$ was pump into the chip at a speed of $1 \mu \mathrm{L} / \mathrm{min}$ for $40 \mathrm{~min}$ and then washed 3 times with PBS. Finally, the fluorescence microscope (Nikon Ti2-U) was used for observation and image J software was used to quantify the fluorescence signal collected on the chip. When the fluorescence signal data was processed, we used the relative fluorescence intensity $I$. $I=\left(I_{a}-I_{b}\right) / t$. $I_{a}$ represents the absolute fluorescence signal on the image, $I_{b}$ represents the background fluorescence signal, and $t$ represents exposure time. When analyzing the data, we used the GraphPad Prism 8 software to perform the Mann-Whitney U test, where a $P$ value of less than 0.05 is considered statistically significant. The result of ultracentrifugation followed by NTA (current golden standard) and that of the ZNI chip (without the need of ultracentrifugation) were compared to verify the accuracy of EV quantification of our device.

\section{Western Blot Analysis of EVs}

The EVs derived from HOS, 143B, U2OS, and MG63 cells were mixed with RIPA to lyse the vesicles and extract the protein. $20 \mu \mathrm{L}$ of each sample was added to a $10 \%$ SDS-PAGE Bis-Tris gel, and then performed electrophoretic separation. Afterwards, the protein on the gel was transferred to the nitrocellulose membrane, and then blocked with 5\% BSA on a shaker at room temperature for $1 \mathrm{~h}$ and washed with TBST 3 times. Anti-CD9 (Abcam), anti-CD81 (Abcam), anti-CD63 (Abcam) and antiVIM (CST) antibodies were added and incubated for $11 \mathrm{~h}$ on a shaker at $4^{\circ} \mathrm{C}$. After being washed 3 times, secondary antibody was added and incubated for $1.5 \mathrm{~h}$ at room temperature. After being washed, the nitrocellulose membrane was exposed and finally completed protein qualitative detection.

\section{Validation of VIM Expression in Public Database}

To validate VIM expression as a potential biomarker of metastatic OS, we retrieved the data of 127 OS patients (the largest dataset in the database) regarding the association of gene expression with the likehood of metastasis from R2 database (35). VIM expression was dichotomized into VIM-high expression and VIM-low expression based on its median value. Kaplan-Meier survival analysis was performed to validate VIM as a biomarker of OS metastasis. 


\section{RESULTS}

\section{Fabrication and Characterization of the ZNI Chip}

The $\mathrm{ZnO}$ growth chip, consisting of 4 channels with a width of 200 $\mu \mathrm{m}$, a height of $75 \mu \mathrm{m}$, and a length of $2 \mathrm{~cm}$ was first reversibly bonded to a glass slide spin-coated with a $\mathrm{ZnO}$ seed layer and then covered with herringbone (HB) micromixer (Figures 2A, B) to produce turbulent flow and minimize the laminar flow in the microchannel. Under the parallel HB micro-structure (Figure 2C), the hexagonal prism structure of zinc oxide wurtzite left an interspace of 10 100 nm between nanowires (Figures 2D, E), exerting a size exclusion-like effect for retaining EVs (Figure 3E) $(36,37)$.

\section{Optimization of the ZNI Chip for EV Capture}

EVs derived from HOS, a commonly used osteosarcoma cell line, as well as 3 replicates of microfluidic chip studies (manufactured in 3 batches) were performed for further optimizing the parameters of the ZNI chip. As expected, HOS-derived EV s exhibited cup- or sphericalshaped morphologies ranging from 30 to $200 \mathrm{~nm}$ (Figure 3A). Among various single or combination of capture antibodies, there is a significant difference in the quantity of the captured EV among each parameter $(\mathrm{F}=16.62, \mathrm{p}<0.05)$. The quantity of the total captured
EVs was found to be highest when CD63+CD81 double antibody was used (Figure 3B). Furthermore, the micro-channel of ZNI chip was frequently clogged at the height of $10 \mu \mathrm{m}$, and the greatest amount of EVs could be captured at the channel height of $20 \mu \mathrm{m}$ (Figure 3C). Similarly, we observed a significant effect of the channel height to the capture result $(\mathrm{F}=20.05, \mathrm{p}<0.05)$. Next, various injection flow rates ranging from $0.5 \mu \mathrm{L} / \mathrm{min}$ to $4 \mu \mathrm{L} / \mathrm{min}$ (Figure 3D) were further optimized. Interestingly, the capture efficiency reached a plateau from $1 \mu \mathrm{L} / \mathrm{min} \sim 2 \mu \mathrm{L} / \mathrm{min}$, and then drastically dropped at the flow rate of $4 \mu \mathrm{L} / \mathrm{min}$ in comparison to other flow rates. The flow rate was also found to significantly affect the EV capture quantity $(\mathrm{F}=11.24, \mathrm{p}<$ 0.05 ). Considering the experimental efficiency, we finalized $2 \mu \mathrm{L} / \mathrm{min}$ as the optimal injection flow rate for the subsequent clinical sample detection.

Using repeated measures ANOVA, we found no statistical significance of the batch effect among the three replications ( $p>0.05)$, suggesting that EV quantification using our microfluidic device was generally reproducible.

\section{Microfluidic-Based Quantification of Cell Line and Plasma EVs}

With the aforementioned parameters of ZNI chip, the captured HOS-derived EVs were observed to be densely bonded to $\mathrm{ZnO}$ -
A

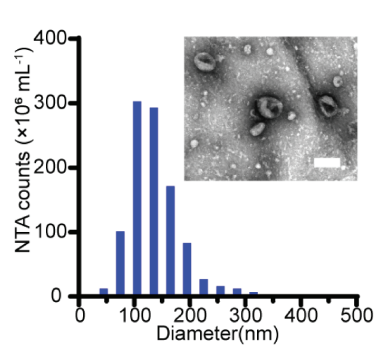

E

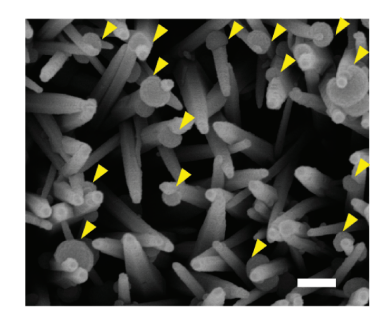

B

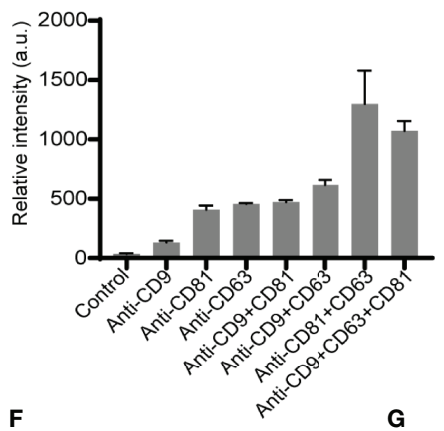

$\mathbf{F}$

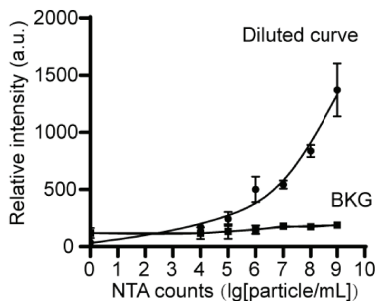

C

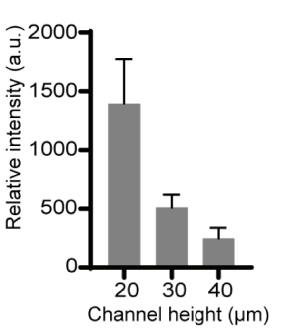

D
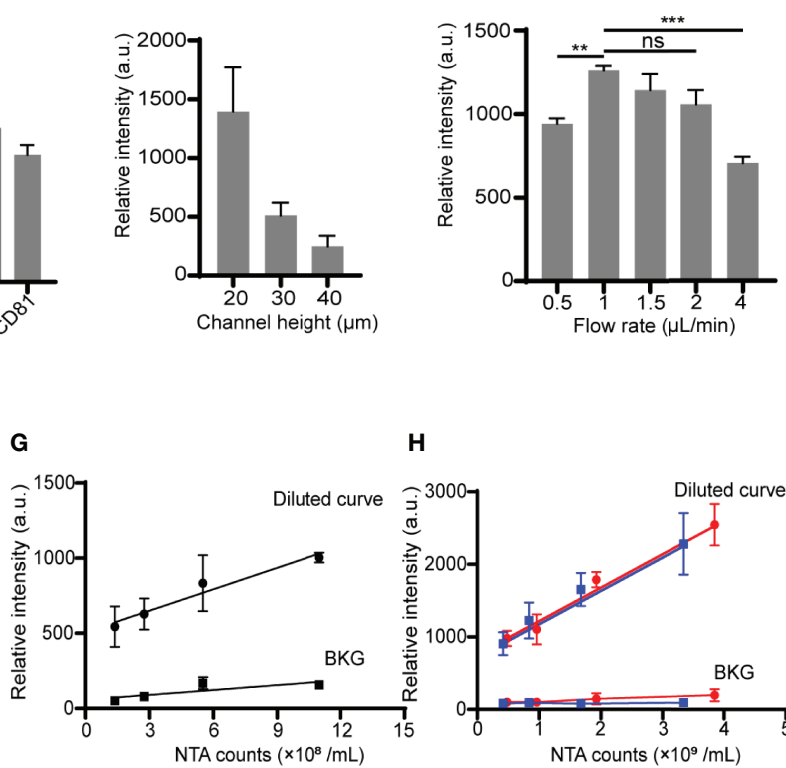

H

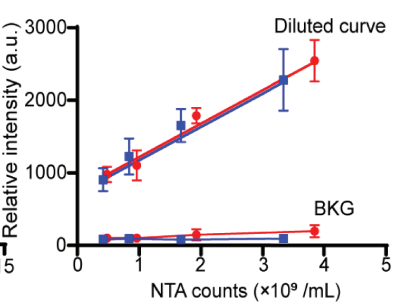

FIGURE 3 | Optimization of the ZNI chip for EV capture. (A)Transmission electron microscope photograph and nanoparticle tracking analysis (NTA) of HOS-derived EVs. Scale bar: 100nm. (B) The effect of various capture antibody combination modes on HOS-derived EV detection efficacy. (C) The effect of various height of the main microfluidic channel of ZNI chip on HOS-derived EV detection efficacy. (D) The effect of various flow velocities ranging from $0.5 \mu \mathrm{L} / \mathrm{min}$ to $4 \mu \mathrm{L} / \mathrm{min}$ on HOS-derived EV detection efficacy. (E) Scanning electron microscope photographs of HOS-derived EVs captured by ZnO nanorods. Scale bar: 150nm. (F) Calibration curves for quantifying total HOS-derived EVs using ZNI chip under optimized function parameters. (G) Correlation of the EVs level between NTA and HOS-derived EVs resuspended in EV-free plasma. (H) Correlation of the EV level between NTA and patient-derived EVs resuspended in EV-deprived plasma using ZNI chip under optimized function parameters. BKG: background. Error bars correspond to the standard deviation $(n=3)$. ns: non-significant, $p>0.05,{ }^{* \star} p<0.01,{ }^{\star \star *} p<0.001$. "Two-way repeated-measures ANOVA" statistical analysis method was used to appraise the significance of different parameters as well as the batch effect in each experiment of (B-D) 
nanorods (Figure 3E). A calibration curve between the fluorescence intensity and the logarithm of the EV concentration from $10^{4}$ particles $/ \mathrm{mL}$ to $10^{9}$ particles $/ \mathrm{mL}$ was obtained, with the limit of detection (LOD) being $\sim 10^{4}$ particles/ $\mathrm{mL}$ in comparison with background signal (ZNI chip without capture antibodies) (Figure 3F). To exclude the potential confounding effect of blood plasma to microfluidic quantification of EVs, HOS- as well as plasma-derived EV resuspension of known concentration was diluted into EV-free plasma into various concentration. Remarkably, we found a robust correlation of the NTA results with the microfluidic EV signal $\left(\mathrm{R}^{2}=0.982 \mathrm{p}=0.009\right)$, but not background signal (ZNI chip without capture antibodies) $\left(\mathrm{R}^{2}=0.725 \mathrm{p}=0.476\right)$ (Figures 3G, $\mathbf{H})$, highlighting the quantification accuracy of our device despite the context of plasma as a complex bio-fluid.

\section{EV Quantification in OS Patients and Healthy Donors}

In order to evaluate the clinical utility of the ZNI chip as a fast screening tool of liquid biopsy, blood samples from OS patients $(n=13)$ and healthy donors $(n=4)$ (Table 1) were used to quantify the total EVs in plasma (Figure 4A). Of the 13 OS samples, 7 were also measured by NTA as a validation. As expected, the fluorescence signal measured on the ZNI chip was highly correlated with NTA results $\left(\mathrm{R}^{2}=0.805 \mathrm{p}<0.05\right)$ (Figure $4 \mathrm{~B}$ and Figure S4). NTA demonstrated that the extracellular vesicle concentration was significantly different between OS patients and the healthy control $\left(1.5 \times 10^{10}\right.$ particles $/ \mathrm{mL}$ vs $5.1 \times 10^{9}$ particles $/ \mathrm{mL}, \mathrm{p}=0.042$ ) (Figure $4 \mathrm{C}$ ), but not between metastasis and non-metastasis patents $\left(1.3 \times 10^{10}\right.$ particles $/ \mathrm{mL}$ vs $1.0 \times 10^{10}$ particles $/ \mathrm{mL}, \mathrm{p}>0.050$ ) (Figure 4D). Consistently, based our ZNI chip, the total EVs were significantly higher in OS patents than the healthy control (1562 a.u. vs 639 a.u.., $\mathrm{p}=0.003$ ) (Figure 4E). The Receiver Operating Characteristic (ROC) curve suggests that our ZNI chip has a favorable diagnostic ability, with an area under curve (AUC) of 0.962 (Figure 4F).

\section{Detection of VIM Positive EVs as a Potential Metastatic Biomarker of OS}

To date, there is no liquid biopsy-based biomarker for the metastasis of OS. We, therefore, sought to investigate the potential utility of a previously reported sarcoma CTC surface marker - VIM (38) - on the captured EVs in our microfluidic chip as a metastatic biomarker of OS. VIM was found to be expressed on the EVs from common OS cell lines such as HOS, 143B, MG63 and U2OS (Figure S6), and the upregulation of VIM in tumor sample was associated with the patients' metastasis-free survival for 127 OS patients in R2 database (35) (Figures 5A, B). We, therefore, quantify the VIM expression status on the captured EVs in our device. Surprisingly, we observed fluorescent co-localization of the VIM (red) with total EVs (green) on the ZNI chip (Figures 5C and S5), while adding DiO/Alexa Fluor 647 to ZNI chip without EV capture antibodies yielding no or minimal fluorescence (negative control), excluding the possibility of nonspecific adsorption of fluorescent dyes (Figure S7). Furthermore, the fluorescent intensity of VIM and total EVs tremendously varied among OS patients (Figure 5D). Although there was no significant difference in total EVs between metastasis and non-metastasis subgroup (Figure 5E), the fluorescent signal of VIM as well as VIM/total EVs ratio were significantly higher in the metastasis compared to the non-metastatic group (Figures 5F, G). The ROC analysis suggested that VIM and VIM/DiO ratio could be a diagnostic biomarker for OS metastasis (Figure $\mathbf{5 H}$ ). The statistical data of VIM and $\mathrm{DiO}$ relative fluorescent intensity from every specimen of this research is displayed in the Supplementary Table.

\section{DISCUSSION}

To our knowledge, there were no previous studies focusing on the optimization and validation of detecting OS-derived EVs

TABLE 1 | Clinical information of osteosarcoma (OS) patients and healthy donors (HD) enrolled in this study.

\begin{tabular}{|c|c|c|c|c|c|}
\hline Patient No. & Gender & Age (year) & Histological Subtype & Clinical Stage & Prognosis \\
\hline P01 & Female & 11 & Conventional OS & $\| I \mathrm{~B}$ & Metastasis \\
\hline P02 & Male & 62 & Conventional OS & IIIB & Metastasis \\
\hline P03 & Male & 8 & Conventional OS & $\| \mathrm{A}$ & Metastasis \\
\hline P04 & Male & 20 & Conventional OS & $\| \mathrm{A}$ & Metastasis \\
\hline P05 & Female & 19 & Periosteal OS & IIIB & Metastasis \\
\hline P06 & Male & 8 & Telangiectatic OS & IIIB & Metastasis \\
\hline P07 & Male & 25 & Conventional OS & $\| \mathrm{IB}$ & Metastasis \\
\hline P08 & Female & 9 & Conventional OS & $\| \mathrm{IB}$ & Metastasis \\
\hline P09 & Male & 15 & Conventional OS & $\mathrm{IB}$ & Non-metastasis \\
\hline P10 & Male & 17 & Conventional OS & $\mid A$ & Non-metastasis \\
\hline P11 & Male & 14 & Conventional OS & $\mathrm{IB}$ & Non-metastasis \\
\hline P12 & Male & 13 & Conventional OS & IB & Non-metastasis \\
\hline P13 & Female & 17 & Conventional OS & $\| \mathrm{A}$ & Non-metastasis \\
\hline HD01 & Male & 25 & $\mathrm{~N} / \mathrm{A}$ & $\mathrm{N} / \mathrm{A}$ & N/A \\
\hline HD02 & Male & 24 & $\mathrm{~N} / \mathrm{A}$ & $\mathrm{N} / \mathrm{A}$ & N/A \\
\hline HD03 & Male & 25 & $\mathrm{~N} / \mathrm{A}$ & $\mathrm{N} / \mathrm{A}$ & N/A \\
\hline HDO4 & Male & 26 & $\mathrm{~N} / \mathrm{A}$ & $\mathrm{N} / \mathrm{A}$ & N/A \\
\hline
\end{tabular}



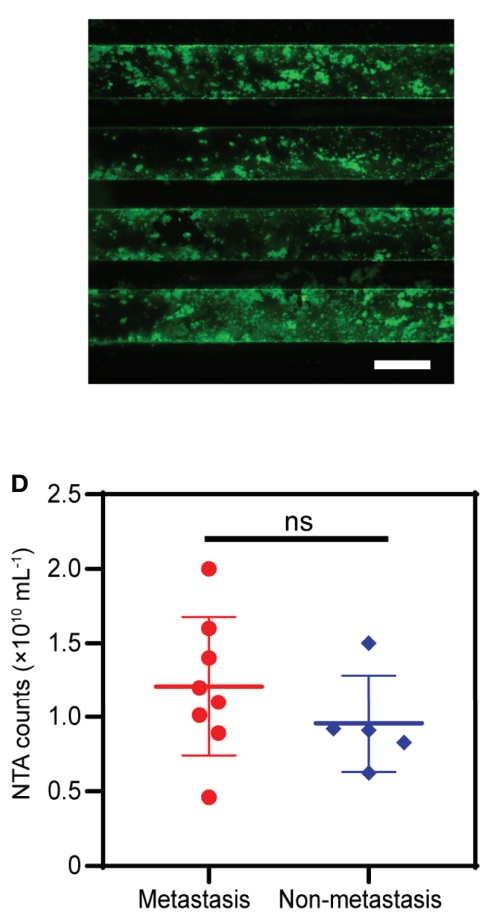

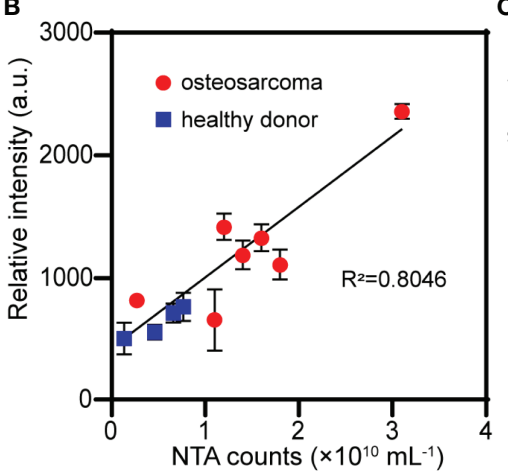

C

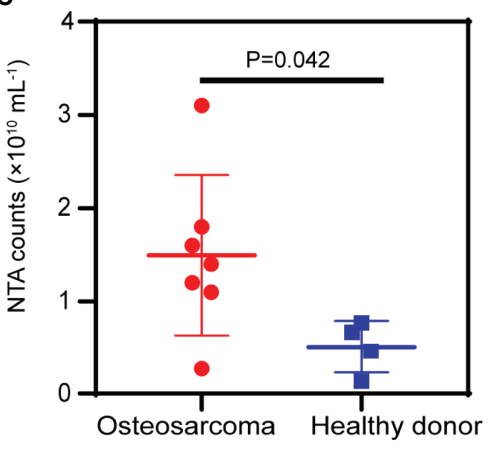

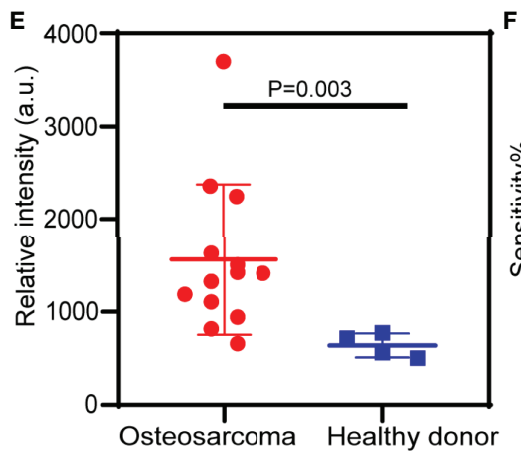

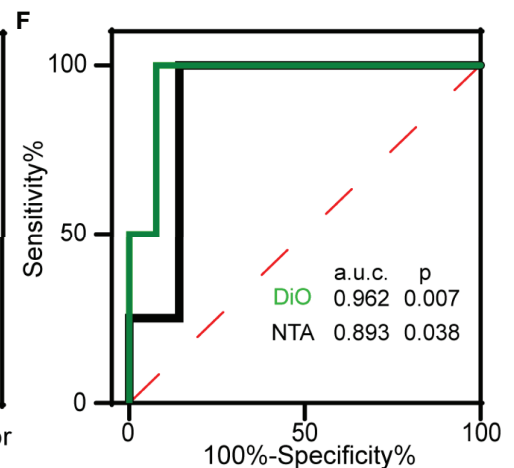

FIGURE 4 | Clinical validation of the ZNI chip in patients of osteosarcoma (OS). (A) Fluorescence image of EVs from plasma captured by ZNI chip. Scale bar: 200 4 m. (B) Correlation analysis of NTA and ZNI chip fluorescence quantification of plasma EVs in 7 OS patients and 4 healthy donors. (C) NTA of the plasma EVs from OS patients and healthy donors. (D) NTA of the plasma EVs from OS patients with metastasis and without metastasis. (E) Fluorescent quantitation of plasma EVs from OS patients and healthy donors. (F) Receiver operating characteristic (ROC) analysis of plasma EVs quantified by ZNI chip and NTA between OS patients and healthy donors. The statistical difference of the two groups was compared using the Mann-Whitney $U$ test. ns, non-significant.

using a microfluidic approach. We, for the first time, demonstrated a microfluidic chip integrated with $\mathrm{ZnO}$ nanorods, which could fast and effectively separate and quantify EVs in the plasma sample of OS patients. By optimizing the functioning parameters based on OS-derived EVs, we have further improved the detection limit to as low as $1.1 \times 10^{4}$ particles $/ \mathrm{mL}$, which is drastically lower than previously reported (26), and the plasma concentration of EV (9). Excellent reproducibility of ZNI chip reveals the immense potential of clinical translation.

Furthermore, our device exceeded previous microfluidicbased isolation techniques in several aspects. Microfluidic chips based on size filtration, acoustic field, and electric field have been widely used in the separation and purification of EVs. Dong et al. developed an ExoID-Chip, which can achieve high-efficiency separation and sensitive detection of EVs through filtration (1). $\mathrm{Wu}$ et al. developed an acoustofluidic platform for EVs (39). In addition, Ibsen et al. used alternating current electrokinetic microarray chips to quickly extract EVs from human plasma (40). However, the EVs obtained by these methods were contaminated with non-vesicular particles, which affected subsequent biological analysis (15). Therefore, the isolation method based on immunoaffinity is more reasonable. Chen et al. presented a $\mathrm{ZnO}$ nanowire chip for immunocapture and colorimetric detection of EVs. However, the chip manufacturing time is long $(>12 \mathrm{~h})$ and the detection process is complicated (26). Our ZNI chip not only greatly reduces the manufacturing time $(<5 \mathrm{~h})$, but also has simple detection steps and greatly improves the limit of detection $\left(1.1 \times 10^{4}\right.$ particles $\left./ \mathrm{mL}\right)$.

Currently, the liquid biopsy for the surveillance of tumor recurrence in OS remains still lacking. Through the analysis of clinical samples, our report was the first one to exploit the possibility of quantifying total extracellular vesicle to distinguish OS patients from healthy controls and identifying OS patients with metastasis from those without based on EV membrane VIM expression. Remarkably, our device could simultaneously quantify EVs and EV membrane biomarker in one single run, which only requires $50 \mu \mathrm{L}$ of archived or fresh plasma samples. In addition, unlike traditional ultracentrifugation as a time-consuming process, it only took about 2 hours from sample collection to signal acquisition. Therefore, our ZNI chip could serve as a portable and efficient tool of liquid biopsy for total EV isolation and high-sensitivity biomarker detection for OS and could be easily adapted for EV membrane biomarker detection for 


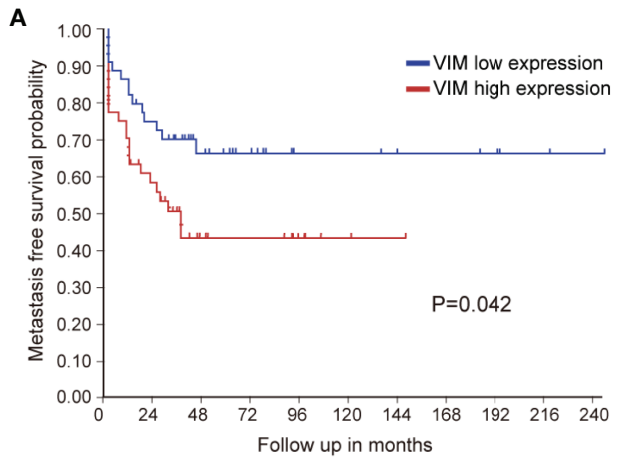

B

C
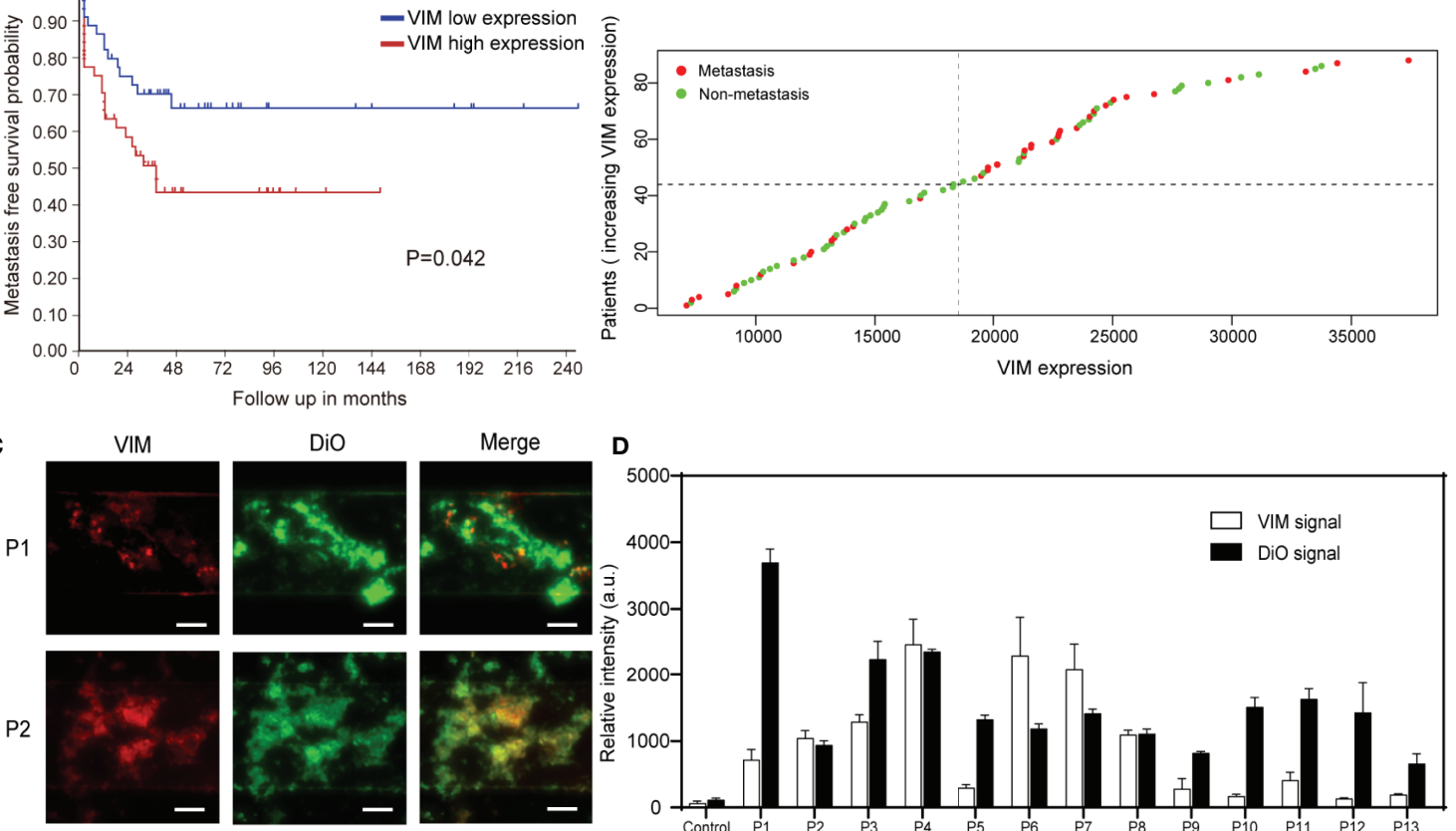

$\mathrm{P} 2$
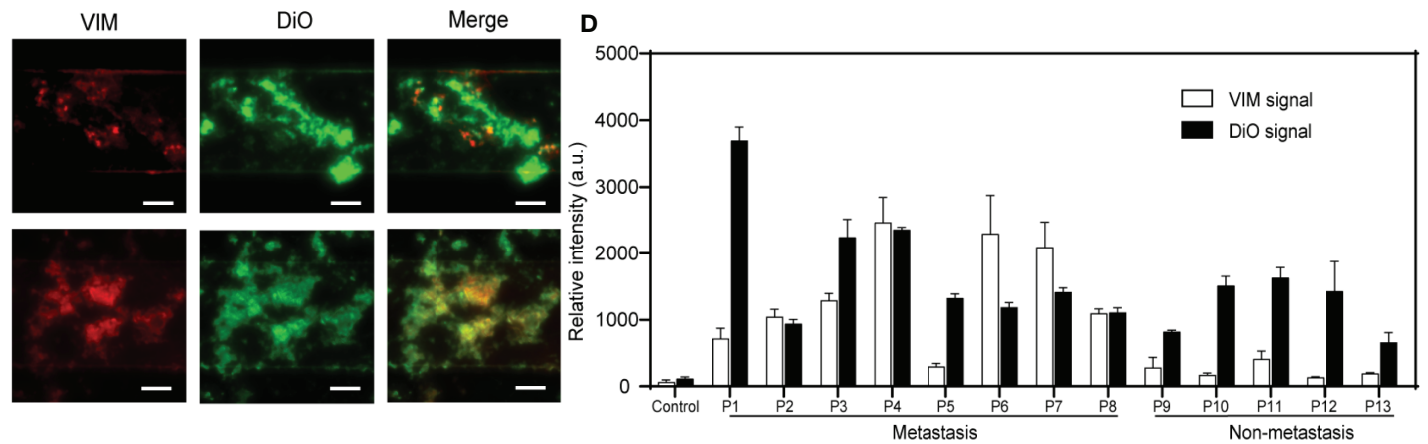

E

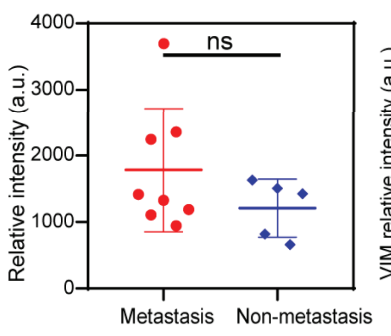

$\mathbf{F}$

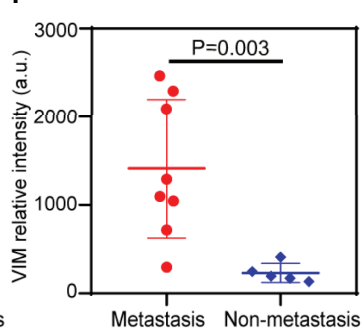

G

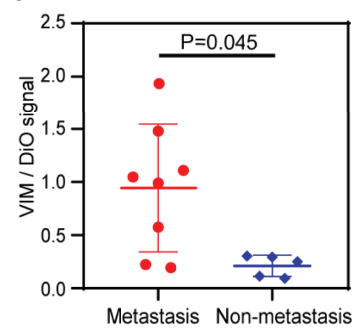

H

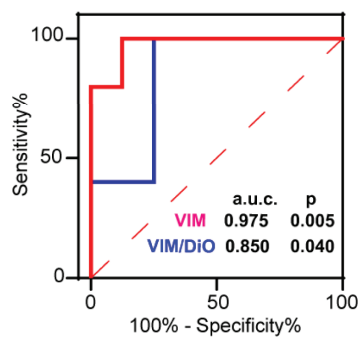

FIGURE 5 | Detection of total EVs and VIM-positive EVs for the surveillance of OS metastasis. (A) Metastasis-free survival probability curve of OS patients with VIM high expression and low expression. (B) Scatter diagram of VIM expression of OS patients with and without metastasis. (C) Exemplary images of immunofluorescence of total EVs and VIM-positive EVs in 2 plasma samples. Scale bar: $100 \mu \mathrm{m}$. (D) Quantification of the EV membrane VIM directly from the plasma of the metastatic OS patients $(n=8)$ and non-metastatic patients $(n=5)$. Error bars indicate $\mathbf{s}$.d. ( $n=3)$. (E) Fluorescence quantification of the total EVs in OS patients with and without metastasis. ns, non-significant. (F, G) The VIM fluorescence signal and the VIM/DiO fluorescence ratio of the plasma EVs derived from OS patients with and without pulmonary metastasis. The statistical difference of the two groups was compared using the Mann-Whitney $U$ test. (H) ROC analysis of VIM absolute signal and VIM/DiO ratio between the metastasis group and the non-metastasis group.

other malignancies. The accuracy of our device might be further improved by introducing a standard sample with a known EV concentration as a control for minimizing the inter-assay variability for further applications. In general, our study suggests that the microfluidic detection of plasma EV membrane biomarker is a promising liquid biopsy technique in the diagnosis and treatment of OS.

\section{DATA AVAILABILITY STATEMENT}

The raw data supporting the conclusions of this article will be made available by the authors, without undue reservation.

\section{ETHICS STATEMENT}

The studies involving human participants were reviewed and approved by Ruijin Hospital Ethics Committee. Written informed consent to participate in this study was provided by the participants' legal guardian/next of kin.

\section{AUTHOR CONTRIBUTIONS}

Y-QX: Conceptualization, Methodology, Investigation, Writing original draft. Q-YB: Methodology, Writing - original draft. S$\mathrm{XY}$ : Investigation. QL: Investigation. YX: Conceptualization. XL: Conceptualization. Y-JL: Conceptualization, Methodology, 
Writing - review and editing, Supervision, Funding acquisition. Y-HS: Conceptualization, Methodology, Writing - review and editing, Supervision, Funding acquisition. All authors contributed to the article and approved the submitted version.

\section{FUNDING}

This work was supported by the National Natural Science Foundation of China (NSFC, Grant Nos. 81773298 and

\section{REFERENCES}

1. Dong X, Chi J, Zheng L, Ma B, Li Z, Wang S, et al. Efficient Isolation and Sensitive Quantification of Extracellular Vesicles Based on an Integrated ExoID-Chip Using Photonic Crystals. Lab Chip (2019) 19(17):2897-904. doi: $10.1039 /$ c9lc00445a

2. Kanwar SS, Dunlay CJ, Simeone DM, Nagrath S. Microfluidic Device (ExoChip) for On-Chip Isolation, Quantification and Characterization of Circulating Exosomes. Lab Chip (2014) 14(11):1891-900. doi: 10.1039/ c4lc00136b

3. Zhang P, Zhou X, He M, Shang Y, Tetlow AL, Godwin AK, et al. Ultrasensitive Detection of Circulating Exosomes With a 3DNanopatterned Microfluidic Chip. Nat BioMed Eng (2019) 3(6):438-51. doi: 10.1038/s41551-019-0356-9

4. Filipazzi P, Bürdek M, Villa A, Rivoltini L, Huber V. Recent Advances on the Role of Tumor Exosomes in Immunosuppression and Disease Progression. Semin Cancer Biol (2012) 22(4):342-9. doi: 10.1016/j.semcancer.2012.02.005

5. Hoshino A, Costa-Silva B, Shen TL, Rodrigues G, Hashimoto A, Tesic Mark $\mathrm{M}$, et al. Tumour Exosome Integrins Determine Organotropic Metastasis. Nature (2015) 527(7578):329-35. doi: 10.1038/nature15756

6. Poggio M, Hu T, Pai CC, Chu B, Belair CD, Chang A, et al. Suppression of Exosomal PD-L1 Induces Systemic Anti-Tumor Immunity and Memory. Cell (2019) 177(2):414-27. doi: 10.1016/j.cell.2019.02.016

7. Tkach M, Théry C. Communication by Extracellular Vesicles: Where We Are and Where We Need to Go. Cell (2016) 164(6):1226-32. doi: 10.1016/ j.cell.2016.01.043

8. Shao H, Im H, Castro CM, Breakefield X, Weissleder R, Lee H. New Technologies for Analysis of Extracellular Vesicles. Chem Rev (2018) 118 (4):1917-50. doi: 10.1021/acs.chemrev.7b00534

9. van der Pol E, Böing AN, Harrison P, Sturk A, Nieuwland R. Classification, Functions, and Clinical Relevance of Extracellular Vesicles. Pharmacol Rev (2012) 64(3):676-705. doi: 10.1124/pr.112.005983

10. Yáñez-Mó M, Siljander PR, Andreu Z, Zavec AB, Borràs FE, Buzas EI, et al. Biological Properties of Extracellular Vesicles and Their Physiological Functions. J Extracell Vesicles (2015) 4:27066. doi: 10.3402/jev.v4.27066

11. Caradec J, Kharmate G, Hosseini-Beheshti E, Adomat H, Gleave M, Guns E. Reproducibility and Efficiency of Serum-Derived Exosome Extraction Methods. Clin Biochem (2014) 47(13-14):1286-92. doi: 10.1016/ j.clinbiochem.2014.06.011

12. Chen C, Skog J, Hsu CH, Lessard RT, Balaj L, Wurdinger T, et al. Microfluidic Isolation and Transcriptome Analysis of Serum Microvesicles. Lab Chip (2010) 10(4):505-11. doi: 10.1039/b916199f

13. He M, Crow J, Roth M, Zeng Y, Godwin AK. Integrated Immunoisolation and Protein Analysis of Circulating Exosomes Using Microfluidic Technology. Lab Chip (2014) 14(19):3773-80. doi: 10.1039/c4lc00662c

14. Zhao Z, Yang Y, Zeng Y, He M. A Microfluidic ExoSearch Chip for Multiplexed Exosome Detection Towards Blood-Based Ovarian Cancer Diagnosis. Lab Chip (2016) 16(3):489-96. doi: 10.1039/c5lc01117e

15. Jeppesen DK, Fenix AM, Franklin JL, Higginbotham JN, Zhang Q, Zimmerman LJ, et al. Reassessment of Exosome Composition. Cell (2019) 177(2):428-45.e18. doi: 10.1016/j.cell.2019.02.029

16. Peng C, Wang J, Bao Q, Wang J, Liu Z, Wen J, et al. Isolation of Extracellular Vesicle With Different Precipitation-Based Methods Exerts a Tremendous Impact on the Biomarker Analysis for Clinical Plasma Samples. Cancer
31870978); Shanghai Science and Technology Committee (Grant No. 17411951900) and Clinical Research Plan of SHDC (Grant No. SHDC2020CR3078B).

\section{SUPPLEMENTARY MATERIAL}

The Supplementary Material for this article can be found online at: https://www.frontiersin.org/articles/10.3389/fonc.2021.709255/ full\#supplementary-material

Biomarkers: Section A Dis Markers (2020) 29(3):373-85. doi: 10.3233/cbm201651

17. Rider MA, Hurwitz SN, Meckes DGJr. ExtraPEG: A Polyethylene GlycolBased Method for Enrichment of Extracellular Vesicles. Sci Rep (2016) 6:23978. doi: 10.1038/srep23978

18. Tian Y, Gong M, Hu Y, Liu H, Zhang W, Zhang M, et al. Quality and Efficiency Assessment of Six Extracellular Vesicle Isolation Methods by NanoFlow Cytometry. J Extracell Vesicles (2020) 9(1):1697028. doi: 10.1080/ 20013078.2019.1697028

19. Han S, Xu Y, Sun J, Liu Y, Zhao Y, Tao W, et al. Isolation and Analysis of Extracellular Vesicles in a Morpho Butterfly Wing-Integrated Microvortex Biochip. Biosens Bioelectron (2020) 154:112073. doi: 10.1016/j.bios.2020.112073

20. Kang YT, Hadlock T, Jolly S, Nagrath S. Extracellular Vesicles on Demand (EVOD) Chip for Screening and Quantification of Cancer-Associated Extracellular Vesicles. Biosens Bioelectron (2020) 168:112535. doi: 10.1016/ j.bios.2020.112535

21. Lo TW, Zhu Z, Purcell E, Watza D, Wang J, Kang YT, et al. Microfluidic Device for High-Throughput Affinity-Based Isolation of Extracellular Vesicles. Lab Chip (2020) 20(10):1762-70. doi: 10.1039/c9lc01190k

22. Wan Y, Maurer M, He HZ, Xia YQ, Hao SJ, Zhang WL, et al. Enrichment of Extracellular Vesicles With Lipid Nanoprobe Functionalized Nanostructured Silica. Lab Chip (2019) 19(14):2346-55. doi: 10.1039/c8lc01359d

23. Xu H, Liao C, Zuo P, Liu Z, Ye BC. Magnetic-Based Microfluidic Device for On-Chip Isolation and Detection of Tumor-Derived Exosomes. Anal Chem (2018) 90(22):13451-8. doi: 10.1021/acs.analchem.8b03272

24. Chen GD, Fachin F, Colombini E, Wardle BL, Toner M. Nanoporous MicroElement Arrays for Particle Interception in Microfluidic Cell Separation. Lab Chip (2012) 12(17):3159-67. doi: 10.1039/c2lc40109f

25. Chen GD, Fachin F, Fernandez-Suarez M, Wardle BL, Toner M. Nanoporous Elements in Microfluidics for Multiscale Manipulation of Bioparticles. Small (2011) 7(8):1061-7. doi: 10.1002/smll.201002076

26. Chen Z, Cheng SB, Cao P, Qiu QF, Chen Y, Xie M, et al. Detection of Exosomes by ZnO Nanowires Coated Three-Dimensional Scaffold Chip Device. Biosens Bioelectron (2018) 122:211-6. doi: 10.1016/j.bios.2018.09.033

27. Kim J, Li Z, Park I. Direct Synthesis and Integration of Functional Nanostructures in Microfluidic Devices. Lab Chip (2011) 11(11):1946-51. doi: $10.1039 / \mathrm{c} 1 \mathrm{lc} 20079 \mathrm{~h}$

28. Suwatthanarak T, Thiodorus IA, Tanaka M, Shimada T, Takeshita D, Yasui T, et al. Microfluidic-Based Capture and Release of Cancer-Derived Exosomes via Peptide-Nanowire Hybrid Interface. Lab Chip (2021) 21(3):597-607. doi: 10.1039/d0lc00899k

29. Arya SK, Saha S, Ramirez-Vick JE, Gupta V, Bhansali S, Singh SP. Recent Advances in $\mathrm{ZnO}$ Nanostructures and Thin Films for Biosensor Applications: Review. Anal Chim Acta (2012) 737:1-21. doi: 10.1016/j.aca.2012.05.048

30. Guo L, Shi Y, Liu X, Han Z, Zhao Z, Chen Y, et al. Enhanced Fluorescence Detection of Proteins Using $\mathrm{ZnO}$ Nanowires Integrated Inside Microfluidic Chips. Biosens Bioelectron (2018) 99:368-74. doi: 10.1016/j.bios.2017.08.003

31. Hao N, Zhang M, Zhang JXJ. Microfluidics for ZnO Micro-/Nanomaterials Development: Rational Design, Controllable Synthesis, and On-Chip Bioapplications. Biomater Sci (2020) 8(7):1783-801. doi: 10.1039/c9bm01787a

32. Stott SL, Hsu CH, Tsukrov DI, Yu M, Miyamoto DT, Waltman BA, et al. Isolation of Circulating Tumor Cells Using a Microvortex-Generating Herringbone-Chip. Proc Natl Acad Sci U.S.A. (2010) 107(43):18392-7. doi: $10.1073 /$ pnas.1012539107 
33. Stroock AD, Dertinger SK, Ajdari A, Mezic I, Stone HA, Whitesides GM. Chaotic Mixer for Microchannels. Science (2002) 295(5555):647-51. doi: 10.1126/science.1066238

34. Wang S, Esfahani M, Gurkan UA, Inci F, Kuritzkes DR, Demirci U. Efficient on-Chip Isolation of HIV Subtypes. Lab Chip (2012) 12(8):1508-15. doi: $10.1039 / \mathrm{c} 2 \mathrm{lc} 20706 \mathrm{k}$

35. Koster J, Volckmann R, Zwijnenburg D, Molenaar P, Versteeg R. Abstract 2490: R2: Genomics Analysis and Visualization Platform. Cancer Res (2019) 79(13 Supplement):2490. doi: 10.1158/1538-7445.AM2019-2490

36. Wang Z, Wu HJ, Fine D, Schmulen J, Hu Y, Godin B, et al. Ciliated Micropillars for the Microfluidic-Based Isolation of Nanoscale Lipid Vesicles. Lab Chip (2013) 13(15):2879-82. doi: 10.1039/c3lc41343h

37. Xia Y, Tang Y, Yu X, Wan Y, Chen Y, Lu H, et al. Label-Free Virus Capture and Release by a Microfluidic Device Integrated With Porous Silicon Nanowire Forest. Small (2017) 13(6):1603135. doi: 10.1002/smll.201603135

38. Satelli A, Mitra A, Cutrera JJ, Devarie M, Xia X, Ingram DR, et al. Universal Marker and Detection Tool for Human Sarcoma Circulating Tumor Cells. Cancer Res (2014) 74(6):1645-50. doi: 10.1158/0008-5472.can-13-1739

39. Wu M, Ouyang Y, Wang Z, Zhang R, Huang PH, Chen C, et al. Isolation of Exosomes From Whole Blood by Integrating Acoustics and Microfluidics. Proc Natl Acad Sci U.S.A. (2017) 114(40):10584-9. doi: 10.1073/pnas.1709210114
40. Ibsen SD, Wright J, Lewis JM, Kim S, Ko SY, Ong J, et al. Rapid Isolation and Detection of Exosomes and Associated Biomarkers From Plasma. ACS Nano (2017) 11(7):6641-51. doi: 10.1021/acsnano.7b00549

Conflict of Interest: The authors declare that the research was conducted in the absence of any commercial or financial relationships that could be construed as a potential conflict of interest.

Publisher's Note: All claims expressed in this article are solely those of the authors and do not necessarily represent those of their affiliated organizations, or those of the publisher, the editors and the reviewers. Any product that may be evaluated in this article, or claim that may be made by its manufacturer, is not guaranteed or endorsed by the publisher.

Copyright (c) $2021 \mathrm{Xu}, \mathrm{Bao}, \mathrm{Yu}, \mathrm{Liu}, \mathrm{Xie}, \mathrm{Li}$, Liu and Shen. This is an open-access article distributed under the terms of the Creative Commons Attribution License (CC BY). The use, distribution or reproduction in other forums is permitted, provided the original author(s) and the copyright owner(s) are credited and that the original publication in this journal is cited, in accordance with accepted academic practice. No use, distribution or reproduction is permitted which does not comply with these terms. 